\title{
Evaluation of Gastrointestinal Patient Reported Outcomes Measurement Information System (GI-PROMIS) Symptom Scales in Subjects With Inflammatory Bowel Diseases
}

\author{
Bharati Kochar, MD, MSCR ${ }^{1}$, Christopher F. Martin, MSPH ${ }^{1}$, Michael D. Kappelman, MD, MPH ${ }^{1}$ \\ Wenli Chen, MS, MA ${ }^{1}$, Robert S. Sandler, MD, MPH ${ }^{1}$ and Millie D. Long, MD, MPH ${ }^{1}$ \\ OBJECTIVES: Patient reported outcomes (PROs) are important treatment endpoints in inflammatory bowel diseases \\ (IBD). We evaluated the gastrointestinal (GI) PRO Measurement Information System (PROMIS) in \\ IBD subjects.
}

METHODS: $\quad$ Crohn's and Colitis Foundation of America's Partners is an Internet-based cohort of IBD subjects. Participants complete surveys, including demographics, disease characteristics, PROMIS domains, disease activity (short Crohn's disease activity index or simple clinical colitis activity index) and quality of life (QoL) indices. In a nested cross-sectional study, we used univariate and bivariate analyses to assess associations between 8 GI-PROMIS domains (reflux, swallowing, diarrhea, nausea, belly pain, gas, incontinence, and constipation) and QoL and disease activity indices.

RESULTS: $\quad$ The study included 2,378 Crohn's Disease (CD) and 1,455 ulcerative colitis (UC) respondents with a median age of 41 years. Median disease duration was 11 years for CD subjects and 8 years for UC subjects; $57 \%$ of CD subjects and $42 \%$ of UC subjects were in remission. Among symptomatic CD subjects, those with active CD reported significantly worse symptoms on all 8 domains than those in remission. The same was observed for UC subjects with the exception of disrupted swallowing. IBD subjects with worse QoL reported significantly worse symptoms on all 8 domains compared to those with better QoL.

CONCLUSIONS: In IBD subjects experiencing GI symptoms, GI-PROMIS domains were strongly associated with disease activity and QoL indices. GI-PROMIS holds potential as PRO measures in IBD and correlates with other validated indices in this population.

Am J Gastroenterol 2018; 113:72-79; doi:10.1038/ajg.2017.240; published online 29 August 2017

\section{INTRODUCTION}

In patients with inflammatory bowel diseases (IBD), such as Crohn's disease (CD) and ulcerative colitis (UC), common symptoms include diarrhea, abdominal pain, or rectal bleeding (1-4). However, other gastrointestinal and psychosocial symptoms may be present, particularly when disease is active. Many of these additional symptoms impact patients physically and emotionally and have a negative impact on quality of life $(5,6)$. These symptoms are legitimate targets for treatments. Various symptom scales have been studied to assess the efficacy of IBD treatment (7-10). The Food and Drug Administration (FDA) has determined that for clinical trials of IBD treatments, co-primary endpoints of patient reported outcomes (PROs) and endoscopic endpoints are required. While candidate PRO measures have been proposed, a complete inventory of GI symptoms using validated PRO scales has not been available.

IBD patients are reported to commonly have psychosocial symptoms such as depression and anxiety $(11,12)$. Results of one

${ }^{1}$ Center for Gastrointestinal Biology \& Disease, University of North Carolina, Chapel Hill, North Carolina, USA; ${ }^{2}$ Division of Gastroenterology, Cedars-Sinai Medical Center, Los Angeles, California, USA. Correspondence: Millie D. Long, MD, MPH, Division of Gastroenterology and Hepatology, University of North Carolina, Campus Box \#7080, 130 Mason Farm Road, Chapel Hill, North Carolina 27599-7080, USA. E-mail: millie_long@med.unc.edu

Received 21 January 2017; accepted 26 June 2017 
prospective cohort study suggest that depressive symptoms may increase the risk for CD among women (13). Additionally, depression has been shown to impact the course of IBD and is associated with worsened disease activity and complications (14). Furthermore, psychological symptoms can pose treatment barriers (15). However, there are no data to indicate how specifi c gastrointestinal symptoms correlate with psychosocial symptoms in patients with IBD.

PROs are important factors in understanding the burden of IBD. Th ere are a full range of GI symptoms that contribute to the burden of IBD. Th e National Institutes of Health's Patient Reported Outcomes Measurement Information System (PROMIS) is a publically available, standardized set of PROs that cover physical, mental and social health. Recently, eight scales were developed for GI symptom domains under this framework (16). The re i s a greater need for the use of PROs to advance the understanding of symptoms in IBD patients. PROs can also be used both in clinical practice and in clinical trials as important endpoints. In this study, we aimed to determine whether GI-PROMIS domains can be used to assess symptoms in IBD patients.

\section{METHODS}

\section{Study setting}

Th e Crohn's and Colitis Foundation of America (CCFA) Partners study is a longitudinal Internet-based cohort of people living with IBD. Th e development of this cohort has been described in detail previously (17). In brief, we recruited participants with a self-reported diagnosis of $\mathrm{CD}$, UC or indeterminate colitis (IC) who were 18 years of age or older through CCFA e-mail rosters, websites, various social media outlets, and at educational and fundraising events. To date, the cohort has enrolled over 15,000 individuals with IBD. All participants completed a baseline survey including demographic information, questions about their IBD history, symptoms and medication use. Respondents then complete bi-annual surveys to update disease and demographic information. CCFA Partners surveys include a number of validated IBD activity indices, quality of life measures and PROMIS assessments as described below.

\section{Instruments}

Th e GI-PROMIS scales assess 8 domains: gastroesophageal refl ux (13 items), disrupted swallowing (7 items), diarrhea (5 items), bowel incontinence/soilage (4 items), nausea and vomiting (4 items), constipation (9 items), belly pain (6 items), and gas/ bloat/fl atulence (12 items) (16). All scales are reported in percentiles, among individuals who report that symptom.

Th e psychosocial PROMIS scales assess 6 domains: anxiety (4 items), depressive symptoms (4 items), fatigue (4 items), pain interference (4 items), social satisfaction (4 items) and sleep disturbance (4 items)( 18 ). All scales are calibrated using an item response theory graded response model and scored on a T-score metric with a mean of 50 and standard deviation (s.d.) of 10 in the US general population. A higher score denotes more symptoms on that scale, with the exception of social satisfaction, where a lower score indicates better social satisfaction. Based on prior data in chronic diseases, a minimally important difference (MID) for the psychosocial scales is $2-3$ points (19).

Disease activity instruments were also completed by participants at the time of the PROMIS scales. Subjects with CD completed the short Crohn's Disease Activity Index (sCDAI); on this scale, remission is defined as a score $<150$. Ulcerative colitis or indeterminate colitis subjects completed the Simple Clinical Colitis Activity Index (SCCAI); remission is defined as a score $\leq 2$. The short Inflammatory Bowel Disease Questionnaire (SIBDQ) is a measure of quality of life, a lower score indicates a worse quality of life.

\section{Study design}

We performed a cross-sectional analysis of IBD subjects enrolled in CCFA Partners from January 2015 to April 2016 who answered GI and psychosocial PROMIS domains. We stratified subjects by factors such as surgical status, disease activity, and disease duration. We also assessed PROMIS domains by classes of medications.

\section{Statistical analysis}

We used descriptive statistics and bivariate analyses to evaluate GI-PROMIS measures relative to validated instruments such as the SCDAI, SCCAI and SIBDQ $(20,21)$. We conducted a sensitivity analysis including only UC patients who have not undergone surgery. All analyses were performed in STATA 14.0 (College Station, TX). The study protocol was approved by the institutional review board at the University of North Carolina at Chapel Hill.

\section{RESULTS}

\section{Demographics}

There were 2,378 subjects with CD (Table 1a) and 1,455 subjects with UC (Table 1b; the IC patients were included with UC), who completed GI-PROMIS questionnaires. Respondents were predominantly female and white: $74 \%$ of CD and $72 \%$ of UC subjects were female; $95 \%$ of CD subjects and $92 \%$ of UC subjects were white. A total of $72 \%$ of CD subjects and $77 \%$ of UC subjects reported a college degree or greater. The median age was 41 years for CD subjects (interquartile range (IQR): 29-54) and UC subjects (IQR: 31-53). Median disease duration was 11 years (IQR: 4-22) for CD subjects and 8 years (IQR: 3-15) for UC subjects. Most of the respondents had previously been hospitalized: $82 \%$ of $\mathrm{CD}$ subjects and $62 \%$ of UC subjects. Half of the CD subjects and $11 \%$ of UC subjects had previous surgery for IBD. At the time of the questionnaire, $57 \%$ of CD subjects and $42 \%$ of UC subjects were in remission.

\section{GI-PROMIS in subjects with Crohn's disease}

Among CD respondents, 24\% reported reflux, 9\% reported difficulty swallowing, 52\% reported diarrhea, $44 \%$ reported nausea and vomiting, $43 \%$ reported belly pain, 59\% reported gas, $16 \%$ reported bowel incontinence and 15\% reported constipation (Figure 1). Symptomatic CD subjects reported a higher level of symptoms than symptomatic subjects in the general population 
Table 1a. Characteristics of the population with Crohn's disease

\begin{tabular}{|c|c|}
\hline Characteristic & $\begin{array}{c}\text { Crohn's disease, }(n=2,378) \\
\% \text { or median (IQR) }\end{array}$ \\
\hline$\%$ Female & 74 \\
\hline Age (years) & $41(29-54)$ \\
\hline \multicolumn{2}{|l|}{ Race } \\
\hline$\%$ White & 95 \\
\hline$\%$ Black & 1 \\
\hline$\%$ College graduate or greater & 72 \\
\hline Body mass index (BMI) & $24(22-29)$ \\
\hline$\%$ Current smokers & 7 \\
\hline Disease duration (years) & $11(4-22)$ \\
\hline \multicolumn{2}{|l|}{$\%$ Currently used medications } \\
\hline Corticosteroids & 13 \\
\hline Mesalamine agents & 26 \\
\hline Immunomodulators & 29 \\
\hline Anti-TNF Biologicals & 49 \\
\hline Probiotics & 26 \\
\hline Antibiotics & 4 \\
\hline Narcotics & 12 \\
\hline \% With a prior IBD hospitalization & 82 \\
\hline$\%$ With prior IBD surgery & 50 \\
\hline Disease activity (\% remissiona) & 57 \\
\hline SIBDQ & $5.0(3.9-5.8)$ \\
\hline
\end{tabular}

IBD, inflammatory bowel diseases; IQR, interquartile range; SIBDQ, short inflammatory bowel disease questionnaire; TNF, tumor necrosis factor.

${ }^{a}$ Defined as a short Crohn's disease activity index (sCDAl) $<150$.

(54th percentile for diarrhea, 57th percentile for nausea and vomiting, 55th percentile for belly pain, 53rd percentile for gas, 56th percentile for bowel incontinence and 53rd percentile for constipation; Figure 2).

\section{GI-PROMIS in subjects with ulcerative colitis}

Among UC respondents, 9\% reported reflux, 3\% reported diffi culties swallowing, $17 \%$ reported diarrhea, $8 \%$ reported nausea and vomiting, $19 \%$ reported belly pain, $27 \%$ reported gas, $6 \%$ reported bowel incontinence and $7 \%$ reported constipation (Figure 1). Symptomatic UC subjects reported similar degrees of symptoms compared to symptomatic subjects in the general population (50th percentile for diarrhea, 49th percentile for belly pain and 48th percentile for constipation; Figure 2). Similar trends were seen in a sub-group analysis of UC subjects who did not have surgery.

\section{Differences by surgical status}

Compared to CD subjects who did not have prior surgery for $C D$, those who had surgery reported more diarrhea $(P<0.01)$, belly pain $(P=0.02)$, gas $(P=0.04)$ and bowel incontinence $(P<0.01)$.
Table 1b. Characteristics of the population with ulcerative colitis

\begin{tabular}{|c|c|}
\hline Characteristic & $\begin{array}{c}\text { Ulcerative colitis, ( } n=1455) \\
\% \text { of median (IQR) }\end{array}$ \\
\hline$\%$ Female & 72 \\
\hline Age (years) & $41(31-53)$ \\
\hline \multicolumn{2}{|l|}{ Race } \\
\hline$\%$ White & 92 \\
\hline$\%$ Black & 1 \\
\hline$\%$ College graduate or greater & 77 \\
\hline Body mass index (BMI) & 25 (22-29) \\
\hline$\%$ Current smokers & 3 \\
\hline Disease Duration (years) & $8(3-15)$ \\
\hline \multicolumn{2}{|l|}{$\%$ Currently Used Medications } \\
\hline Corticosteroids & 13 \\
\hline Mesalamine agents & 59 \\
\hline Immunomodulators & 22 \\
\hline Anti-TNF Biologicals & 26 \\
\hline Probiotics & 31 \\
\hline Antibiotics & 3 \\
\hline Narcotics & 6 \\
\hline \% With a prior IBD hospitalization & 62 \\
\hline$\%$ With prior IBD surgery & 11 \\
\hline Disease activity (\% remission ${ }^{a}$ ) & 42 \\
\hline SIBDQ & $5.2(4.1-5.9)$ \\
\hline
\end{tabular}

IBD, inflammatory bowel diseases; IQR, interquartile range; SIBDQ, short inflammatory bowel disease questionnaire; TNF, tumor necrosis factor.

aDefined as a Simple Clinical Colitis Activity Index (SCCAI) $<2$.

There were no meaningful differences between UC subjects based on surgical status.

\section{Differences by disease activity, quality of life and psychosocial measures}

Among symptomatic CD subjects, those with active disease reported significantly more symptoms on all 8 GI-PROMIS domains than those in remission (Table 2a). With the exception of disrupted swallowing, symptomatic UC subjects with active disease also reported significantly more symptoms on the other 7 GI-PROMIS than UC subjects in remission (Table $2 \mathbf{b}$ ).

When stratified by quartiles of the SIBDQ, symptomatic CD and UC subjects in the lowest quartile (worst quality of life) reported significantly more symptoms on all 8 GI-PROMIS domains compared to those in the highest quartile (best quality of life). There was a trend toward decreasing GI symptoms with higher SIBDQ scores (Table 3).

Respondents who reported more symptoms on the GI-PROMIS domains reported significantly more symptoms on the psychosocial PROMIS domains. We present data from three representative domains, diarrhea, belly pain and gas. Amongst CD subjects, 


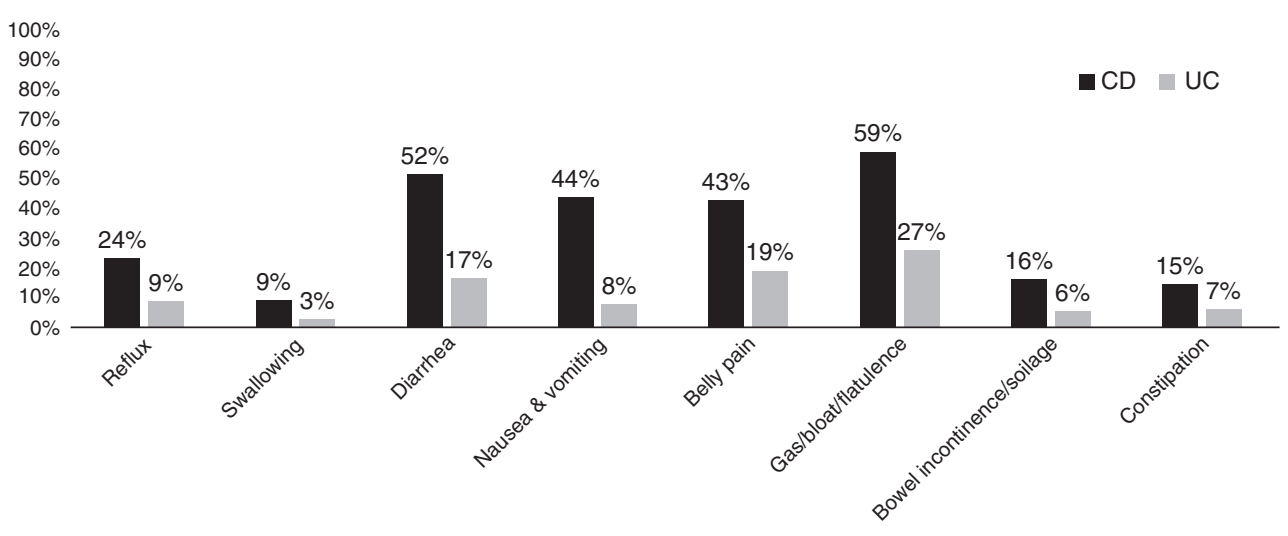

Figure 1. Prevalence of gastrointestinal symptoms in patients with inflammatory bowel diseases (IBD) as measured by GI-PROMIS scales.

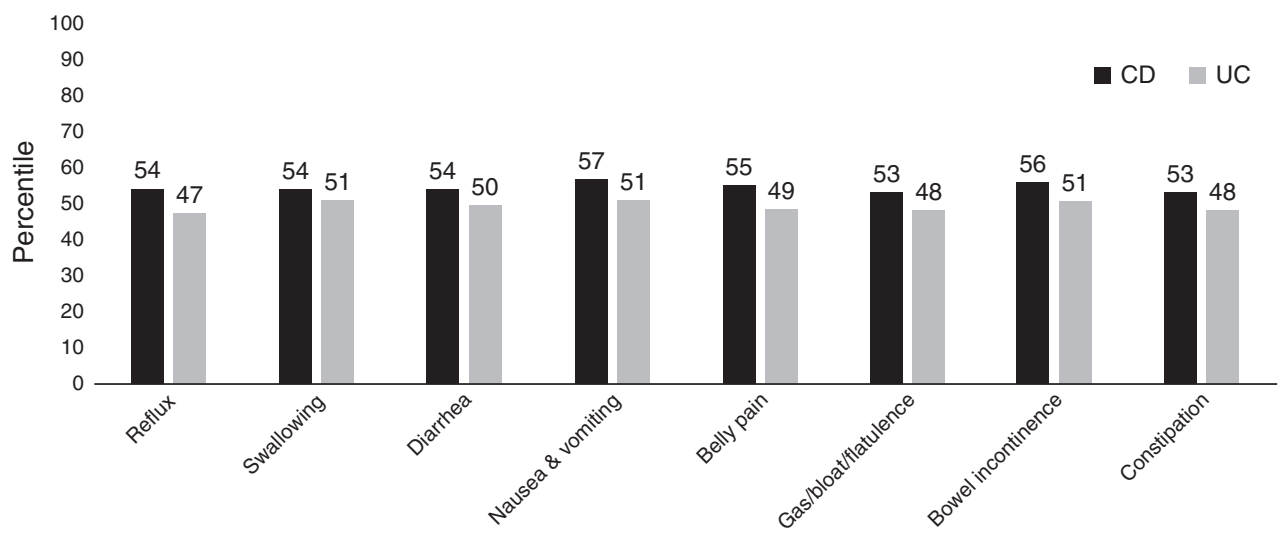

Figure 2. Gastrointestinal Patient Reported Outcome Measurement Information System (GI-PROMIS) symptom scales in symptomatic subjects with inflammatory bowel disease (domains are reported as percentiles compared to all those in the general population who reported that symptom).

Table 2a. Gastrointestinal Patient Reported Outcome Measurement Information System (GI-PROMIS) domain percentiles stratified by remission status for symptomatic subjects with Crohn's disease

$\begin{array}{lccc} & \begin{array}{c}\text { Active disease } \\ (\boldsymbol{n}=\mathbf{1 , 0 2 3})\end{array} & \begin{array}{c}\text { Remission } \\ (\boldsymbol{n}=\mathbf{1 , 3 5 5 )}\end{array} & \boldsymbol{P} \text {-value } \\ \text { Reflux } & 63 & 44 & <0.01 \\ \text { Swallowing } & 59 & 46 & <0.01 \\ \text { Diarrhea } & 67 & 40 & <0.01 \\ \text { Nausea and vomiting } & 63 & 42 & <0.01 \\ \text { Belly pain } & 68 & 37 & <0.01 \\ \text { Gas/bloat/flatulence } & 67 & 42 & <0.01 \\ \text { Bowel incontinence } & 63 & 44 & <0.01 \\ \text { Constipation } & 64 & 48 & <0.01\end{array}$

Remission in Crohn's disease defined as a short Crohn's Disease Activity Index (sCDAl) $<150$.

in general, those who had more GI symptoms reported signifi -cantly more psychosocial symptoms than those who had less GI symptoms compared to those who did not report any GI symp-
Table 2b. Gastrointestinal Patient Reported Outcome Measurement Information System (GI-PROMIS) domain percentiles stratified by remission status for symptomatic subjects with ulcerative colitis

$\begin{array}{lccc} & \begin{array}{c}\text { Active disease } \\ (\boldsymbol{n}=\mathbf{8 4 4})\end{array} & \begin{array}{c}\text { Remission } \\ (\boldsymbol{n}=\mathbf{6 1 1})\end{array} & \boldsymbol{P} \text {-value } \\ \text { Reflux } & 50 & 36 & <0.01 \\ \text { Swallowing } & 51 & 39 & 0.09 \\ \text { Diarrhea } & 58 & 26 & <0.01 \\ \text { Nausea and vomiting } & 52 & 37 & <0.01 \\ \text { Belly pain } & 55 & 33 & <0.01 \\ \text { Gas/bloat/flatulence } & 55 & 39 & <0.01 \\ \text { Bowel incontinence } & 54 & 29 & <0.01 \\ \text { Constipation } & 56 & 36 & <0.01\end{array}$

Remission in ulcerative colitis defined as Short Clinical Colitis Activity Index (SCCAI) $<2$.

toms (Table 4a). In the UC cohort, those who had more GI symptoms reported significantly more psychosocial symptoms than those who had less GI symptoms; however, those who had less GI 
Table 3. Gastrointestinal Patient Reported Outcome Measurement Information System (GI-PROMIS) percentiles in symptomatic inflammatory bowel disease subjects stratified by quartiles of the Short Inflammatory Bowel Disease Questionnaire (SIBDQ)

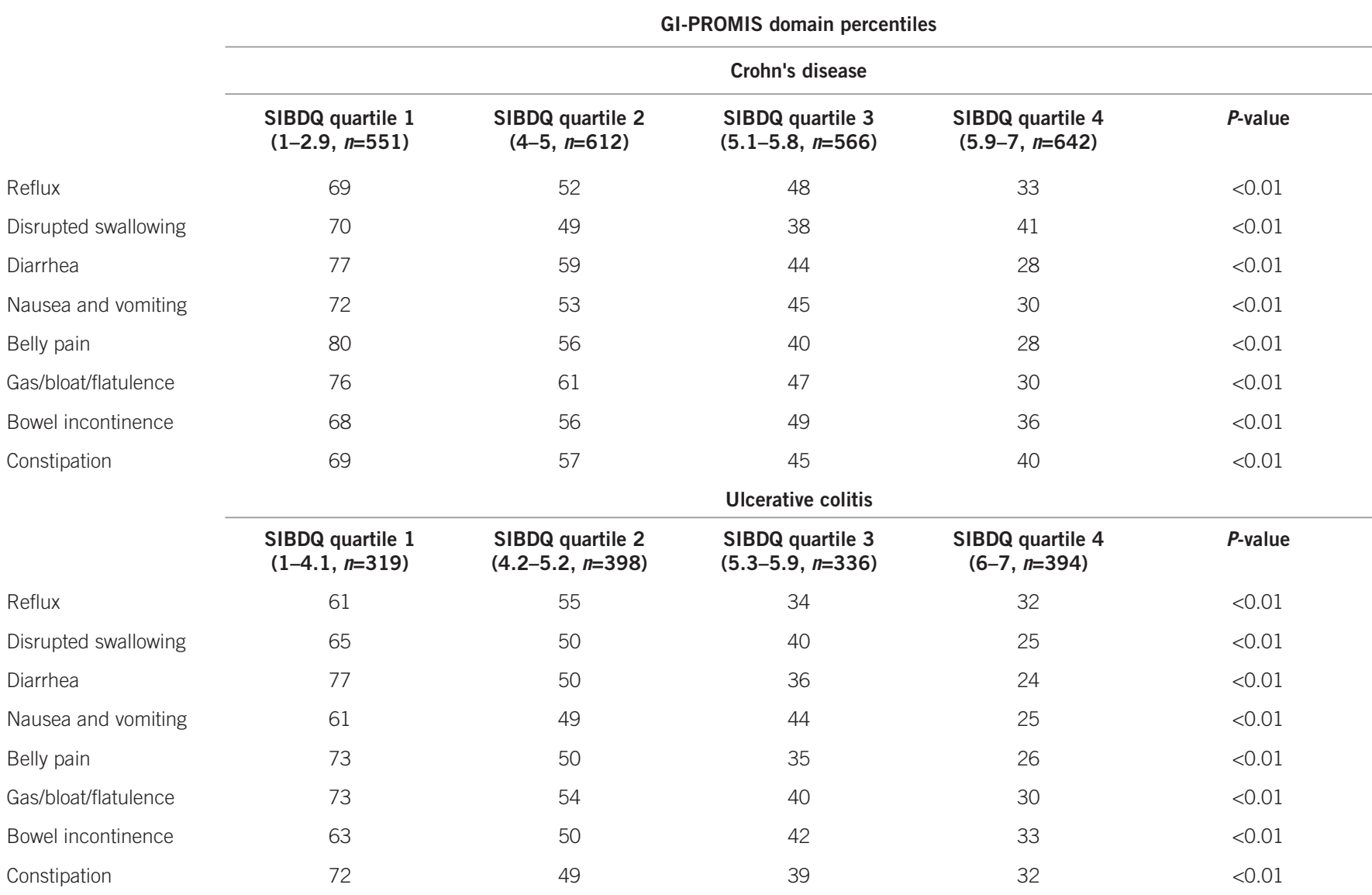

GI-PROMIS percentiles cannot be calculated in persons not experiencing a given symptom.

symptoms reported psychosocial symptoms in the same range as those who did not have any GI symptoms (Table $4 \mathbf{b}$ ).

\section{Differences by narcotic use}

$S$ ymptomatic CD subjects on narcotics reported signifi cantly more symptoms on all 8 GI-PROMIS domains compared to those not on narcotics. Symptomatic UC subjects on narcotics reported more refl ux (68th percentile vs. 45th percentile, $P \varangle 0.01$ ), darrhea (71st percentile vs. 48th percentile, $P<0.01$ ), nausea and vomiting (66th percentile vs. 48th percentile, $P<0.01$ ), belly pain (65th percentile vs. 47 th percentile, $P<0.01$ ) and gas (69th percentile vs. 47 th percentile, $P<0.01)$ compared to those not on narcotics.

\section{DISCUSSION}

Patient reported outcomes (PROs) are an essential component of patient centered research. The GI-PROMIS scales are a recent addition to the PROMIS portfolio that are freely available to the public free of charge, low burden for patients to complete and capable of implementation in clinical practice (22). We present data on GI-PROMIS scales in a large cohort of subjects with
IBD. Within the IBD population, there were strong associations between worsened GI symptoms and worsened quality of life, disease activity and psychosocial symptoms.

There have been few studies comparing the prevalence of gastrointestinal symptoms in IBD subjects to those of the general population $(23,24)$. We found that symptomatic IBD subjects report GI symptoms in the same range as the general population who report these symptoms. In essence, regardless of the cause of the symptom (such as diarrhea), subjects with IBD and those without IBD report a similar symptom burden. There were no notable differences when stratified by age, gender or disease duration. It is possible that IBD subjects are accustomed to GI symptoms and have been able to accommodate over time. This finding may also reflect disease control. A sub-group analysis confirmed that those who had active disease reported worsened GI symptoms when compared to those in remission, with the exception of disrupted swallowing in UC subjects. These findings are in line with a prior study demonstrating a high symptom burden in IBD patients. This study also demonstrated that while many patients with inactive disease report GI symptoms, the symptoms burden was highest amongst patients with active IBD (25). 
Table 4a. Association between gastrointestinal symptoms and psychosocial Patient Reported Outcome Measurement Information System (PROMIS) domains in Crohn's disease

Psychosocial PROMIS Domains

GI-PROMIS Domain

$\begin{array}{ccc}\text { No diarrhea } & \text { Low diarrhea } & \text { High diarrhea } \\ (n=578) & (n=569) & (n=536)\end{array}$

Anxiety

50

50
49
54
50
52
51
Low belly
ain ( $n=465$ )

Depressive symptoms

Fatigue

Pain interference

Sleep disturbance

Social Satisfaction

\section{Anxiety}

Depressive symptoms

Fatigue

Sleep disturbance

Social satisfaction

48

48

52

52
No belly pain
$(n=748)$

49

48

50

52

53

\section{No gas}

$(n=380)$

50

Anxiety

Depressive symptoms

Fatigue

Pain interference

Sleep disturbance

Social satisfaction

57

55

62

58

52

43

High belly

pain $(n=460)$

52
50
55
52
50
Low gas
(n=647)

50

48

52

48

52

52

56

55

61

57

52

45

58
56
64
52
42
High gas
$(n=635)$

The numbers in the table are T scores. The psychosocial PROMIS domains are scored on a T-score metric with a mean of 50 and standard deviation (s.d.) of 10 in the United States general population. A higher score denotes more symptoms on that scale, with the exception of social satisfaction, where a lower score indicates better social satisfaction. A minimally important difference (MID) for the psychosocial scales is 2-3.

Low and high gastrointestinal (GI) symptoms divided at the median reported on the GI-PROMIS scales.

I $t$ is notable that IBD subjects report levels of gastrointestinal symptoms similar to the general population in all domains, including those not traditionally associated with IBD, such as refl $\mathrm{ux}$ and disrupted s wallowing. Prior r esearch has shown that people with medically unexplained symptoms, such as an ulcerative colitis patient who has refl ux or a Crohn's patient who has disrupted swallowing, report more symptoms when recalling a 7 day period compared to people who do not have as many medically unexplained symptoms (26). It is possible that poorly controlled IBD may result in a variety of previously unrecognized symptoms due to a lack of awareness from patients and providers alike. It is also possible that individuals with IBD have a perception of poor overall gastrointestinal health, thus reporting symptoms in all categories.
Table 4b. Association between gastrointestinal symptoms and psychosocial Patient Reported Outcome Measurement Information System (PROMIS) domains in ulcerative colitis

Psychosocial PROMIS

GI-PROMIS domain

Domains

Anxiety
Depressive symptoms
Fatigue
Pain interference
Sleep disturbance
Social Satisfaction

No diarrhea
$(n=533)$
Low diarrhea ( $n=274)$
High diarrhea $(n=206)$

50
48
51
48
52
53
belly pain

51

57

49

55

53

60

$$
50
$$$$
58
$$

51

43

\section{Anxiety}

Depressive symptoms

49

Low belly
pain ( $n=322)$

High belly pain ( $n=212$ )

Fatigue

Sleep disturbance

48

49

52

Social satisfaction

$$
54
$$

No gas

( $n=251$ )

53
50
53
52
50
Low gas
$(n=431)$

57

55

61

52

43

Anxiety

Depressive symptoms

49

47

50

High gas

( $n=317)$

Fatigue

Pain interference

Sleep disturbance

Social satisfaction

5

50

48

52

53

48

56

54

51

59

48

55

52

52

52

46

The numbers in the table are T scores. The psychosocial PROMIS domains are scored on a T-score metric with a mean of 50 and standard deviation (s.d.) of 10 in the United States general population. A higher score denotes more symptoms on that scale, with the exception of social satisfaction, where a lower score indicates better social satisfaction. A minimally important difference (MID) for the psychosocial scales is 2-3.

Low and high gastrointestinal (GI) symptoms divided at the median reported on the GI-PROMIS scales.

Prior studies have shown the association between depression/ anxiety and treatment adherence, worsening symptoms of IBD and general social functioning $(6,15,27,28)$. We demonstrate that those subjects with worsened GI symptoms on the PROMIS scales also report worsened psychosocial symptoms, namely depression, anxiety, pain interference, fatigue, social satisfaction and sleep disturbance. For both CD and UC subjects, minimal levels of GI symptoms did not increase psychosocial symptoms when compared to those without GI symptoms. However, those who have high levels of GI symptoms report psychosocial symptoms that are meaningfully higher than reference populations. In summary, those with well controlled GI symptoms are similar to those who are asymptomatic, but those with poorly controlled GI symptoms have worsened psychosocial symptoms. 
In a sub-group analyses by SIBDQ and disease activity indices, those subjects who reported worsened quality of life and disease activity also reported worsened GI symptoms for all eight GI-PROMIS scales. Th i s s upports the fact $t$ hat $t$ he P ROMIS scales are good measures of GI symptoms in IBD subjects and are responsive to diff erences in disease activity. However, it should be noted that these disease activity measures include symptoms and therefore, it is not surprising that they do correlate so well.

While there are data on the harmful eff e cts of $n$ arcotics in patients with $\operatorname{IBD}(29,30)$, there are no specific reports correlating narcotics with worsening GI symptoms, as we demonstrate. It is possible that use of narcotics is a marker of poorly controlled IBD (31), explaining the presence of worsened GI symptoms. It is also possible that use of narcotics is a marker for poorly controlled symptoms of irritable bowel syndrome (IBS), which can be prevalent in IBD patients $(24,32)$. Finally, it is possible that narcotics themselves could paradoxically worsen various GI symptom measures, perhaps through mechanisms of alternation of motility.

Th ere are several strengths of this study, including the large sample size and geographically diverse population. However, there are limitations as well. Primarily, this is a cross-sectional study, which precludes the ability to determine causality. Th e demographics of the population, predominantly female, white and well-educated, does not refl ect the IBD population across the US. Ther efore, the CCFA-Partners Internet-based cohort is not representative of the US IBD population. Th is cohort focuses on patient reported information without individualized confirmation from a medical record. However, a validation study from a subset of this cohort revealed $97 \%$ concordance between self-reported diagnosis of IBD and evaluation of the medical record (33). In addition, the self-reported data collection should not aff ect the reliability of the responses for the PROMIS scales.

In conclusion, this cross-sectional analysis is the first report of the previously validated GI-PROMIS scales in a large sample of IBD subjects. Th ese measures were found to correlate with other validated scales of quality of life and disease activity. Furthermore, higher percentiles on a GI-PROMIS domains correlate with worse psychosocial PROs in this IBD population. GI-PROMIS domains hold potential as important PRO measures in IBD subjects. Th i $s$ is especially important as there is a need for improved treatment endpoints in clinical IBD management and in IBD clinical trials.

\section{ACKNOWLEDGMENTS}

This research was supported by grants from the Crohn's and Colitis Foundation of America, National Institutes of Health (T32DK07634, P30 DK034987) and the Patient Centered Outcomes Research Institute.

\section{CONFLICT OF INTEREST}

Guarantor of the article: Millie D. Long, MD, MPH. Specific Author Contributions: Bharati Kochar: interpreting data \& drafting the manuscript. Christopher F. Martin: conducting the study, collecting and interpreting data. Michael D. Kappelman: plan-ning/conducting the study, collecting/interpreting data and drafting the manuscript. Brennan M. Spiegel: planning the study. Wenli Chen: collecting the data. Robert S. Sandler: planning the study, drafting the manuscript. Millie D. Long: planning/conducting the study, collecting/interpreting data and drafting the manuscript.

Financial Support: This research was supported by grants from the Crohn's and Colitis Foundation of America, National Institutes of Health (T32DK07634, P30 DK034987) and the Patient Centered Outcomes Research Institute.

Potential competing interests: Michael D. Kappelman: Research support from Abbvie and Janssen, consultant to Abbvie and Janssen. Brennan M. Spiegel: Research support from Astra-Zeneca, Commonwealth Laboratories, GI Logic, Ironwood Pharmaceuticals, Nestle Health Sciences, Novartis, Shire, Takeda; Consultant for Allergan, Commonwealth Laboratories, Ironwood Pharmaceuticals, Synergy Pharmaceuticals; Royalties from GI Logic and My Total Health; Ownership My Total Health. Millie D. Long: Consultant Abbvie, Takeda, Theravance. Pfizer. The remaining authors declare no conflict of interest.

\section{Study Highlights}

\section{WHAT IS CURRENT KNOWLEDGE}

$\checkmark$ Inflammatory bowel diseases (IBD) subjects frequently report diarrhea and abdominal pain.

$\checkmark$ IBD subjects have increased depression and anxiety.

$\checkmark$ Patient reported outcomes (PROs) are important factors in understanding the burden of IBD.

\section{WHAT IS NEW HERE}

Gastrointestinal (GI) symptoms, such as reflux and disrupted swallowing, are also commonly reported in IBD.

Among IBD subjects experiencing GI symptoms, worsened GI symptoms correlate with worsened depression and anxiety.

Among IBD subjects experiencing GI symptoms, narcotic use and corticosteroid use are associated with worsened Gl symptoms.

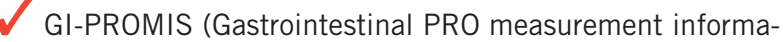
tion system) scales correlate with other validated measures of disease activity and quality of life in IBD.

\section{REFERENCES}

1. Zaharie R, Tantau A, Zaharie F et al. Diagnostic delay in Romanian patients with inflammatory bowel disease: risk factors and impact on the disease course and need for surgery. J Crohns Colitis 2016;10:306-14.

2. Maconi G, Orlandini L, Asthana AK et al. The impact of symptoms, irritable bowel syndrome pattern and diagnostic investigations on the diagnostic delay of Crohn's disease: A prospective study. Dig Liver Dis 2015;47:646-51.

3. Danese S, Fiorino G, Mary JY et al. Development of red flags index for early referral of adults with symptoms and signs suggestive of Crohn's disease: an IOIBD initiative. J Crohns Colitis 2015;9:601-6.

4. Heikenen JB, Werlin SL, Brown CW et al. Presenting symptoms and diagnostic lag in children with inflammatory bowel disease. Inflamm Bowel Dis 1999;5:158-60.

5. Haapamaki J, Turunen U, Roine RP et al. Impact of demographic factors, medication and symptoms on disease-specific quality of life in inflammatory bowel disease. Qual Life Res 2009;18:961-9.

6. Jelsness-Jorgensen LP, Bernklev T, Moum B. Coexisting irritable bowel-like symptoms in inflammatory bowel disease in remission is associated with 
impaired social functioning and increased bodily pain. Gastroenterol Nurs 2014;37:280-7.

7. Sun H, Lee JJ, Papadopoulos EJ et al. Alternate endpoints and clinical outcome assessments in pediatric ulcerative colitis registration trials. J Pediatr Gastroenterol Nutr 2014;58:12-17.

8. Fausel R, Afzali A. Biologics in the management of ulcerative colitiscomparative safety and efficacy of TNF-alpha antagonists. Ther Clin Risk Manag 2015;11:63-73.

9. Bruining DH, Sandborn WJ. Do not assume symptoms indicate failure of anti-tumor necrosis factor therapy in Crohn's disease. Clin Gastroenterol Hepatol 2011;9:395-9.

10. Scholmerich J. Review article: should we treat symptoms or lesions in Crohn's disease? The case for treating symptoms. Aliment Pharmacol Ther 2006;24(Suppl 3):33-6.

11. Mahadev S, Young JM, Selby W et al. Self-reported depressive symptoms and suicidal feelings in perianal Crohn's disease. Colorectal Dis 2012;14:331-5.

12. Pirinen T, Kolho KL, Ashorn M et al. Sleep and emotional and behavioral symptoms in adolescents with inflammatory bowel disease. Sleep Disord 2014;2014:379450.

13. Ananthakrishnan AN, Khalili H, Pan A et al. Association between depressive symptoms and incidence of Crohn's disease and ulcerative colitis: results from the Nurses' Health Study. Clin Gastroenterol Hepatol 2013;11:57-62.

14. Gaines LS, Slaughter JC, Horst SN et al. Association between affectivecognitive symptoms of depression and exacerbation of Crohn's disease. Am J Gastroenterol 2016;111:864-70.

15. Gray WN, Denson LA, Baldassano RN et al. Treatment adherence in adolescents with inflammatory bowel disease: the collective impact of barriers to adherence and anxiety/depressive symptoms. J Pediatr Psychol 2012;37:282-91.

16. Spiegel BM, Hays RD, Bolus R et al. Development of the NIH Patient-Reported Outcomes Measurement Information System (PROMIS) gastrointestinal symptom scales. Am J Gastroenterol 2014;109:1804-14.

17. Long MD, Kappelman MD, Martin CF et al. Development of an internetbased cohort of patients with inflammatory bowel diseases (CCFA Partners): methodology and initial results. Inflamm Bowel Dis 2012;18:2099-106.

18. Kappelman MD, Long MD, Martin CF et al. Evaluation of the patient-reported outcomes measurement information system in a large cohort of patients with inflammatory bowel diseases. Clin Gastroenterol Hepatol 2014;12:1315-23 e2.

19. Yost KJ, Eton DT, Garcia SF et al. Minimally important differences were estimated for six Patient-Reported Outcomes Measurement Information System-Cancer scales in advanced-stage cancer patients. J Clin Epidemiol 2011;64:507-16
20. Irvine EJ, Zhou Q, Thompson AK. The Short Inflammatory Bowel Disease Questionnaire: a quality of life instrument for community physicians managing inflammatory bowel disease. CCRPT Investigators. Canadian Crohn's Relapse Prevention Trial. Am J Gastroenterol 1996;91:1571-8.

21. Thia K, Faubion WA, Loftus EV et al. Short CDAI: development and validation of a shortened and simplified Crohn's disease activity index. Inflamm Bowel Dis 2011;17:105-11.

22. Almario CV, Chey WD, Khanna D et al. Impact of National Institutes of Health Gastrointestinal PROMIS Measures in Clinical Practice: Results of a Multicenter Controlled Trial. Am J Gastroenterol 2016;111:1546-56.

23. Nobrega AC, Ferreira BR, Oliveira GJ et al. Dyspeptic symptoms and delayed gastric emptying of solids in patients with inactive Crohn's disease. BMC Gastroenterol 2012;12:175.

24. Simren M, Axelsson J, Gillberg R et al. Quality of life in inflammatory bowel disease in remission: the impact of IBS-like symptoms and associated psychological factors. Am J Gastroenterol 2002;97:389-96.

25. Singh S, Blanchard A, Walker JR et al. Common symptoms and stressors among individuals with inflammatory bowel diseases. Clin Gastroenterol Hepatol 2011;9:769-75.

26. Houtveen JH, Oei NY. Recall bias in reporting medically unexplained symptoms comes from semantic memory. J Psychosom Res 2007;62:277-82.

27. Camara RJ, Schoepfer AM, Pittet V et al. Swiss Inflammatory Bowel Disease Cohort Study, Group. Mood and nonmood components of perceived stress and exacerbation of Crohn's disease. Inflamm Bowel Dis 2011;17:2358-65.

28. Ghosh S, Mitchell R. Impact of inflammatory bowel disease on quality of life: Results of the European Federation of Crohn's and Ulcerative Colitis Associations (EFCCA) patient survey. J Crohns Colitis 2007;1:10-20.

29. Long MD, Barnes EL, Herfarth $\mathrm{HH}$ et al. Narcotic use for inflammatory bowel disease and risk factors during hospitalization. Inflamm Bowel Dis 2012;18:869-76.

30. Lichtenstein GR, Feagan BG, Cohen RD et al. Serious infections and mortality in association with therapies for Crohn's disease: TREAT registry. Clin Gastroenterol Hepatol 2006;4:621-30.

31. Cross RK, Wilson KT, Binion DG. Narcotic use in patients with Crohn's disease. Am J Gastroenterol 2005;100:2225-9.

32. Palm O, Moum B, Jahnsen J et al. Fibromyalgia and chronic widespread pain in patients with inflammatory bowel disease: a cross sectional population survey. J Rheumatol 2001;28:590-4.

33. Randell RL, Long MD, Cook SF et al. Validation of an internet-based cohort of inflammatory bowel disease (CCFA partners). Inflamm Bowel Dis 2014;20:541-4. 